\title{
ON A FRIEDRICHS EXTENSION RELATED TO UNBOUNDED SUBNORMAL OPERATORS
}

\author{
SAMEER CHAVAN $\dagger$ and AMEER ATHAVALE $\ddagger$ \\ Department of Mathematics, Indian Institute of Technology Bombay, Powai, Mumbai 400076, India \\ †e-mail: sameer@math.iitb.ac.in \\ $\ddagger$-mail:athavale@math.iitb.ac.in
}

(Received 20 January, 2005; accepted 14 October, 2005)

\begin{abstract}
We combine the theory of sectorial sesquilinear forms with the theory of unbounded subnormal operators in Hilbert spaces to characterize the Friedrichs extensions of multiplication operators (with analytic symbols) in certain functional Hilbert spaces. Such characterizations lead to abstract Galerkin approximations and generalized wave equations.
\end{abstract}

2000 Mathematics Subject Classification. Primary 41A65, 47B20. Secondary 35K90, 41A10, 47A07, 47B32.

1. Preliminaries. The theory of sectorial sesquilinear forms is a powerful tool in the study of differential operators (refer to [4] and [6], for example). The present paper is an illustration of the utility of that theory in the study of multiplication operators (with analytic symbols) in certain functional Hilbert spaces. Our methods make an essential use of the fact that the multiplication operators under consideration are unbounded cyclic subnormal operators (see definitions below), and rely crucially on the theory of such operators as expounded in [9]. (See also [7], [8].) The interaction of the theory of sectorial sesquilinear forms with that of unbounded subnormals was explored by the authors in [2] with a special attention paid to those subnormal operators that admit 'analytic models'. While the investigations here are in the same spirit as those carried out in [2], they are in no way dependent on the results obtained in [2]. The main result of the paper (Theorem 3 in Section 2) is the identification of the Friedrichs extension of a certain multiplication operator with a 'maximal' multiplication operator in the relevant functional Hilbert space. Such an identification has natural advantages as demonstrated by a couple of applications in Section 3 (Proposition 3 and Proposition 4). In the present section we record some requisites pertaining to sectorial sesquilinear forms and unbounded subnormals.

For a subset $A$ of the complex plane $\mathcal{C}$, let $A^{*}, \operatorname{int}(A), \bar{A}$ and $A^{c}$ respectively denote the conjugate, the interior, the closure and the complement of $A$ in $\mathcal{C}$. We use $\mathcal{R}$ to denote the real line, and $\operatorname{Re} z$ and $\operatorname{Im} z$ respectively denote the real and imaginary parts of a complex number $z$. Let $\mathcal{H}$ be a complex infinite-dimensional separable Hilbert space with the inner product $\langle\cdot, \cdot\rangle_{\mathcal{H}}$ and the corresponding norm $\|\cdot\|_{\mathcal{H}}$. If $S$ is a densely defined linear operator in $\mathcal{H}$ with domain $\mathcal{D}(S)$, then we use $\sigma(S), \sigma_{p}(S)$, $\sigma_{a p}(S)$ to respectively denote the spectrum, the point spectrum and the approximate point spectrum of $S$. It may be recalled that $\sigma_{p}(S)$ is the set of eigenvalues of $S$, that $\sigma_{a p}(S)$ is the set of those $\lambda$ in $\mathcal{C}$ for which $S-\lambda$ is not bounded below, and that $\sigma(S)$ is the complement of the set of those $\lambda$ in $\mathcal{C}$ for which $(T-\lambda)^{-1}$ exists as a bounded 
linear operator on $\mathcal{H}$. (Refer to Remark 1 below.) For a normal operator $N$ in $\mathcal{H}$, $\sigma(N)=\sigma_{a p}(N)$.

A densely defined linear operator $S$ in $\mathcal{H}$ with domain $\mathcal{D}(S)$ is said to be cyclic if there is a vector $f_{0} \in \mathcal{D}^{\infty}(S)=\cap_{n=0}^{\infty} \mathcal{D}\left(S^{n}\right)($ referred to as a cyclic vector of $S$ ) such that $\mathcal{D}(S)$ is the linear span $\operatorname{lin}\left\{S^{n} f_{0}: n \geq 0\right\}$ of the set $\left\{S^{n} f_{0}: n \geq 0\right\}$.

If $S$ is a densely defined linear operator in $\mathcal{H}$ with domain $\mathcal{D}(S)$, then $S$ is said to be subnormal if there exist a Hilbert space $\mathcal{K}$ containing $\mathcal{H}$ and a densely defined normal operator $N$ in $\mathcal{K}$ with domain $\mathcal{D}(N)$ such that $\mathcal{D}(S) \subset \mathcal{D}(N)$ and $S f=N f, f \in \mathcal{D}(S)$.

If $S$ is a densely defined closable operator in $\mathcal{H}$, then we use $\bar{S}$ to denote the closure of $S$ in $\mathcal{H}$. It is known that every subnormal operator is closable. (See [8].) Let $S$ be a densely defined cyclic subnormal operator in $\mathcal{H}$ with the cyclic vector $f_{0}$. It follows from Proposition 6 of [9] that, for any point $\lambda \in \sigma_{p}\left(S^{*}\right)^{*}$, there exists a unique vector $h_{\lambda}$ in $\mathcal{H}$ such that $p(\lambda)=\left\langle p(S) f_{0}, h_{\lambda}\right\rangle_{\mathcal{H}}$ for every polynomial $p$. One can then define a function $k_{S}$ on $\mathcal{C}$ by setting $k_{S}(\lambda)$ equal to $\left\|h_{\lambda}\right\|^{2}$ if $\lambda \in \sigma_{p}\left(S^{*}\right)^{*}$, and equal to $\infty$ otherwise. If $\operatorname{int}\left(\sigma_{p}\left(S^{*}\right)\right)$ is non-empty and if one defines

$$
\gamma(S)=\left\{\lambda \in \mathcal{C}: k_{S} \text { is finite and continuous in a neighbourhood of } \lambda\right\},
$$

then it follows from Theorem 7 of [9] that $\gamma(S)$ is an open subset of int $\left(\sigma_{p}\left(S^{*}\right)^{*}\right)$ and that $\operatorname{int}\left(\sigma_{p}\left(S^{*}\right)^{*}\right) \backslash \gamma(S)$ is a nowhere dense subset of $\mathcal{C}$; further, as pointed out in Footnote 9 following Theorem 9 of [9], $\sigma(\bar{S}) \backslash \sigma_{a p}(\bar{S}) \subset \gamma(S)$.

Given a Hilbert space $\mathcal{H}$ and a cyclic operator $S$ on $\mathcal{H}$ with a cyclic vector $f_{0}$ of $S$, one can come up with a sequence $r=\left\{r_{n}\right\}_{n \geq 0}$ of complex polynomials such that $e_{n}=r_{n}(S) f_{0}$ and $\operatorname{lin}\left\{e_{n}: n \geq 0\right\}=\operatorname{lin}\left\{S^{n} f_{0}: n \geq 0\right\}$. See [9]. As observed in the proof of Proposition 6 of [9], the polynomials $r_{n}$, so obtained, form a Hamel basis for $\mathcal{C}[z]$, the vector space of complex polynomials in $z$. If $\omega_{r}$ is the set $\left\{z \in \mathcal{C}: \sum_{n=0}^{\infty}\left|r_{n}(z)\right|^{2}<\infty\right\}$, then we define $K_{r}$ on $\omega_{r} \times \omega_{r}$ by $K_{r}(z, w)=\sum_{n=0}^{\infty} \overline{r_{n}(z)} r_{n}(w) \quad\left(z, w \in \omega_{r}\right)$. Since $K_{r}$ is a positive definite kernel on $\omega_{r}$, we can associate with $K_{r}$ a reproducing kernel Hilbert space $\mathcal{H}_{r}$ as described in [1]. The following theorem is Theorem 6 of [9].

TheOREM 1. Suppose that $\mathcal{H}, S, e, r, \omega_{r}$ and $\mathcal{H}_{r}$ are as described in the preceding paragraph. If the point spectrum $\sigma_{p}\left(S^{*}\right)$ of $S^{*}$ is non-empty, then the following are true:

(a) $\mathcal{P}_{r}$, the set of restrictions of members of $\mathcal{C}[z]$ to $\omega_{r}$, is dense in $\mathcal{H}_{r}$;

(b) the operator $M_{z}$ of multiplication by $z$ defined on $\mathcal{P}_{r}$ is cyclic with a cyclic vector the constant polynomial 1 ;

(c) there is a unique partial isometry $W: \mathcal{H} \rightarrow \mathcal{H}_{r}$ with its initial space being the closure of lin $\left\{\sum_{n=0}^{\infty} \overline{r_{n}(\lambda)} e_{n}: \lambda \in \sigma_{p}\left(S^{*}\right)^{*}\right\}$ and its final space being $\mathcal{H}_{r}$ and such that $W S=M_{z} W$

(d) $\omega_{r}=\sigma_{p}\left(S^{*}\right)^{*}=\sigma_{p}\left(M_{z}^{*}\right)^{*}$.

Suppose that, for a cyclic operator $S$ in $\mathcal{H}$ having nonempty point spectrum $\sigma_{p}\left(S^{*}\right)$, the mapping $W$ in (c) of Theorem 1 turns out to be an isometry of $\mathcal{H}$ onto $\mathcal{H}_{r}$; in this case the triple $\left(M_{z}, \mathcal{P}_{r}, \mathcal{H}_{r}\right)$ will be referred to as an analytic model of the cyclic operator $S$.

REMARK 1. It is a practice with some authors (see [5], for example) to define the concept of spectrum for closed linear operators first and then declare the spectrum of a closable linear operator $S$ to be the spectrum of its closure $\bar{S}$; we do not follow that practice in the present paper. Indeed, in keeping with the definition of the spectrum of $S$ as given earlier, we find that Lemma 1 below leads to examples of cyclic subnormal operators $S$ whose spectra are the whole of $\mathcal{C}$, while the spectra of their closures 
are proper subsets of $\mathcal{C}$. Now the proof of Lemma 1 below uses the following fact established in the proof of Theorem 2 of [9]. If $S$ is a closed subnormal operator in $\mathcal{H}$ with a normal extension $N$ acting in $\mathcal{K}$ and $\omega$ is a connected component of $\sigma(N)^{c}$, then either $\omega \cap \sigma(S)=\emptyset$ or $\omega \subset \sigma(S)$. The proof of Theorem 2 of [9] (as presented in [9]) assumes that $\sigma(S)$ equals $\sigma(\bar{S})$ to establish the foregoing assertion also for a non-closed subnormal operator $S$; however, using our definition of the spectrum and without assuming $\sigma(S)=\sigma(\bar{S})$, one can recover the assertion of Theorem 2 of [9] by simply considering two cases: (1) $\sigma(S) \neq \mathcal{C}$ (in which case $S$ is closed) (2) $\sigma(S)=\mathcal{C}$ (in which case $\omega \subset \sigma(S))$.

Let $\mathcal{H}$ be a complex infinite-dimensional separable Hilbert space with the inner product $\langle\cdot, \cdot\rangle_{\mathcal{H}}$ and the corresponding norm $\|\cdot\|_{\mathcal{H}}$. Let $\Gamma$ be a dense subspace of $\mathcal{H}$ such that $\Gamma$ itself is a Hilbert space with the inner product $\langle\cdot, \cdot\rangle_{\Gamma}$ and the corresponding norm $\|\cdot\|_{\Gamma}$. Let there exist a positive number $M_{1}$ satisfying

$$
\|x\|_{\mathcal{H}} \leq M_{1}\|x\|_{\Gamma}, \quad \text { for all } x \in \Gamma .
$$

Let also $\mathcal{F}: \Gamma \times \Gamma \rightarrow \mathcal{C}$ be a sesquilinear form and assume that there exist positive numbers $M_{2}, M_{3}$ and a real number $a$ such that

$$
\begin{gathered}
|\mathcal{F}(x, y)| \leq M_{2}\|x\|_{\Gamma}\|y\|_{\Gamma}, \quad \text { for all } x, y \in \Gamma, \\
\operatorname{Re} \mathcal{F}(x, x) \geq M_{3}\|x\|_{\Gamma}^{2}+a\|x\|_{\mathcal{H}}^{2}, \quad \text { for all } x \in \Gamma .
\end{gathered}
$$

Such $\mathcal{F}$ are referred to as sectorial sesquilinear forms. Corresponding to any such $\mathcal{F}$ we can define a linear operator $A$ in $\mathcal{H}$ as follows: $x \in \mathcal{D}(A)$ if and only if $x \in \Gamma$ and there exists $z$ in $\mathcal{H}$ such that

$$
\mathcal{F}(x, y)=\langle z, y\rangle_{\mathcal{H}}, \quad \text { for all } y \text { in } \Gamma
$$

we set $A x=z$. The linear operator $A$ so defined is referred to as the operator associated with $\mathcal{F}$ and it clearly satisfies

$$
\mathcal{F}(x, y)=\langle A x, y\rangle_{\mathcal{H}}, \quad \text { for all } x \in \mathcal{D}(A) \text { and for all } y \text { in } \Gamma .
$$

For the basic properties of the operator $A$ associated with a sectorial sesquilinear form $\mathcal{F}$, the reader is referred to Chapter 2 of [6]. In particular, it follows from Theorem 2.8.2 in [6] that $A$ is a closed densely defined operator in $\mathcal{H}$ and $\sigma(A)$ is contained in the "truncated cone"

$$
\Lambda=\left\{\zeta \in \mathcal{C}: \operatorname{Re} \zeta \geq a+M_{3} / M_{1}^{2} \text { and }|\operatorname{Im} \zeta| \leq M_{2}(\operatorname{Re} \zeta-a) / M_{3}\right\}
$$

The following result is Theorem 2.12.1 of [6].

THEOREM 2. Suppose that $S$ is a densely defined linear operator in a complex Hilbert space $\mathcal{H}$ such that, for some $r \in \mathcal{R}$ and $M \in(0, \infty)$, we have

$$
\left|\operatorname{Im}\langle S x, x\rangle_{\mathcal{H}}\right| \leq M \operatorname{Re}\langle S x-r x, x\rangle_{\mathcal{H}}, \quad \text { for all } x \in \mathcal{D}(S) .
$$

Then there exist a subspace $\Gamma$ of $\mathcal{H}$, an inner product $\langle\cdot, \cdot\rangle_{\Gamma}$ on $\Gamma$ with the corresponding norm $\|\cdot\|_{\Gamma}$, and a sectorial sesquilinear form $\mathcal{F}$ on $\Gamma$ such that the following assertions hold:

(a) $\mathcal{D}(S)$ is a dense subspace of $\Gamma$ (in the $\|\cdot\|_{\Gamma}$ norm); 
(b) $\langle x, y\rangle_{\Gamma}=(1 / 2)\left(\langle S x, y\rangle_{\mathcal{H}}+\langle x, S y\rangle_{\mathcal{H}}\right)+(1-r)\langle x, y\rangle_{\mathcal{H}}$, for all $x, y \in \mathcal{D}(S)$;

(c) $\mathcal{F}(x, y)=\langle S x, y\rangle_{\mathcal{H}}$, for all $x, y \in \mathcal{D}(S)$.

The linear operator $A$ associated with the sectorial sesquilinear form $\mathcal{F}$ of Theorem 2 is clearly an extension of the operator $S$ and is called the Friedrichs extension of $S$. Referring to the proof of Theorem 2.12.1 in [6] one finds that the constants $M_{1}, M_{2}, M_{3}$ and $a$ corresponding to $\mathcal{F}$ as in (2), (3), (4) are given by $M_{1}=1, M_{2}=$ $M+1+|r-1|, M_{3}=1$ and $a=r-1$; in particular, from (7) we obtain

$$
\sigma(A) \subset \Lambda=\{\zeta \in \mathcal{C}: \operatorname{Re} \zeta \geq r \quad \text { and }|\operatorname{Im} \zeta| \leq(M+1+|r-1|)(\operatorname{Re} \zeta-r+1)\}
$$

2. Multiplication operators with analytic symbols. Let $G$ be an open subset of the complex plane $\mathcal{C}$ and let $w: G \rightarrow(0, \infty)$ be a positive continuous function. We use $\mu_{w}$ to denote the weighted area measure on $G$ defined by $d \mu_{w}(z)=w(z) d z(z \in G)$. Let $L^{2}\left(G, \mu_{w}\right)$ stand for the Hilbert space of $\mu_{w}$-square integrable Lebesgue measurable functions on $G$ (with two functions being identified if they are $\mu_{w}$-almost everywhere equal to each other). Let $L_{a}^{2}\left(G, \mu_{w}\right)=\left\{f \in L^{2}\left(G, \mu_{w}\right): f\right.$ is analytic on $\left.G\right\}$.

As in the case of the Bergman space of a bounded domain (see [3, Ch. 2, Proposition 8.4] and [3, Ch. 2, Theorem 8.5]) it can be verified that $L_{a}^{2}\left(G, \mu_{w}\right)$ is a Hilbert space, and the point evaluation is bounded on $L_{a}^{2}\left(G, \mu_{w}\right)$ so that, for any $\lambda \in G$, there exists $k_{\lambda} \in \mathcal{H}=L_{a}^{2}\left(G, \mu_{w}\right)$ such that

$$
\left\langle f, k_{\lambda}\right\rangle_{\mathcal{H}}=f(\lambda) \text { for all } f \in L_{a}^{2}\left(G, \mu_{w}\right)
$$

Definition 1. A triple $(G, \phi, w)$ is called a weighted analytic domain if $G$ is a nonempty open subset of $\mathcal{C}, \phi: G \rightarrow \mathcal{C}$ is a non-constant analytic function, $w: G \rightarrow(0, \infty)$ is a continuous function, and $\phi^{k} \in L^{2}\left(G, \mu_{w}\right)$, for every non-negative integer $k$. (We interpret $\phi^{0}$ to be the constant function 1 with $1(z)=1$, for all $z$ in $G$.)

Proposition 1. Let $(G, \phi, w)$ be a weighted analytic domain with $P_{\phi}^{2}\left(G, \mu_{w}\right)$ being the closure of $\operatorname{lin}\left\{\phi^{k}: k=0,1,2, \ldots\right\}$ in $L^{2}\left(G, \mu_{w}\right)$. Let $M_{\phi}$ be the operator of multiplication by $\phi$ in $P_{\phi}^{2}\left(G, \mu_{w}\right)$ with $\mathcal{D}\left(M_{\phi}\right)=\operatorname{lin}\left\{\phi^{k}: k=0,1,2, \ldots\right\}$. Then $\phi(G) \subset \sigma_{p}\left(M_{\phi}^{*}\right)^{*}$.

Proof. For any polynomial $p, p \circ \phi \in L_{a}^{2}\left(G, \mu_{w}\right)$. It follows then from (10) that, for any $\lambda \in G$, there exists $k_{\lambda} \in \mathcal{K}=L_{a}^{2}\left(G, \mu_{w}\right)$ such that $\left\langle p \circ \phi, k_{\lambda}\right\rangle_{\mathcal{K}}=(p \circ \phi)(\lambda)$, for every polynomial $p$. Thus $|p(\phi(\lambda))| \leq\left\|k_{\lambda}\right\|_{\mathcal{K}}\|p \circ \phi\|_{\mathcal{K}}=\left\|k_{\lambda}\right\|_{\mathcal{K}}\left\|p\left(M_{\phi}\right) 1\right\|_{\mathcal{H}}$, where $\mathcal{H}=P_{\phi}^{2}\left(G, \mu_{w}\right)$. Since $M_{\phi}$ is a cyclic operator in $P_{\phi}^{2}\left(G, \mu_{w}\right)$ (with cyclic vector 1 ), it follows from Lemma 2 of [9] that $\phi(\lambda) \in \sigma_{p}\left(M_{\phi}^{*}\right)^{*}$.

The operator $M_{\phi}$ in $P_{\phi}^{2}\left(G, \mu_{w}\right)$ corresponding to a weighted analytic domain $(G, \phi, w)$ as described in Proposition 1 is subnormal; indeed, a normal extension of $M_{\phi}$ is $N_{\phi}$ in $L^{2}\left(G, \mu_{w}\right)$ defined by $N_{\phi} f=\phi f$ for $f \in \mathcal{D}\left(N_{\phi}\right)=\left\{f \in L^{2}\left(G, \mu_{w}\right): \phi f \in\right.$ $\left.L^{2}\left(G, \mu_{w}\right)\right\}$. We define $M_{\phi}^{\max }$ to be the operator of multiplication by $\phi$ on $\mathcal{D}\left(M_{\phi}^{\max }\right)=$ $\left\{f \in P_{\phi}^{2}\left(G, \mu_{w}\right): \phi f \in P_{\phi}^{2}\left(G, \mu_{w}\right)\right\}$. It is easy to check that $M_{\phi}^{\max }$ is a closed linear operator in $P_{\phi}^{2}\left(G, \mu_{w}\right) ; M_{\phi}^{\max }$ obviously extends $\overline{M_{\phi}}$. A triple $(G, \phi, w)$ will be called a subdomain of a weighted analytic domain $\left(G^{\prime}, \phi^{\prime}, w^{\prime}\right)$ if $G$ is a non-empty open subset of $G^{\prime}, \phi=\phi^{\prime} / G$, and $w=w^{\prime} / G$. 
Proposition 2. Let $(G, \phi, w)$ be a weighted analytic domain. If $\phi(G)$ is contained in the cone $\Lambda_{r, M}=\{z \in \mathcal{C}:|\operatorname{Im} z| \leq M(\operatorname{Re} z-r)\}$, where $r \in \mathcal{R}$ and $M \in(0, \infty)$, then there exist a subspace $\Gamma$ of $P_{\phi}^{2}\left(G, \mu_{w}\right)$, an inner product $\langle\cdot, \cdot\rangle_{\Gamma}$ on $\Gamma$ with the corresponding norm $\|\cdot\|_{\Gamma}$, and a sectorial sesquilinear form $\mathcal{F}$ on $\Gamma$ such that the following assertions hold:

(a) $\mathcal{D}\left(M_{\phi}\right)$ is a dense subspace of $\Gamma$ (in the $\|\cdot\|_{\Gamma}$ norm);

(b) $\langle f, g\rangle_{\Gamma}=\int_{G}(\operatorname{Re} \phi(z)-r+1) f(z) \overline{g(z)} d \mu_{w}(z)$, for all $f, g \in \mathcal{D}\left(M_{\phi}\right)$;

(c) $\mathcal{F}(f, g)=\int_{G} \phi(z) f(z) \overline{g(z)} d \mu_{w}(z)$, for all $f, g \in \mathcal{D}\left(M_{\phi}\right)$.

Proof. Note that $S=M_{\phi}$ is a densely defined linear operator in $\mathcal{H}=P_{\phi}^{2}\left(G, \mu_{w}\right)$. Also, for any $f$ in $\operatorname{lin}\left\{\phi^{k}: k=0,1,2, \ldots\right\}$, one has, using $\phi(G) \subset \Lambda_{r, M}$, that

$$
\begin{aligned}
\left|\operatorname{Im}\left\langle M_{\phi} f, f\right\rangle_{\mathcal{H}}\right| & =\left|\operatorname{Im} \int_{G} \phi(z) f(z) \overline{f(z)} d \mu_{w}(z)\right| \\
& \leq \int_{G}|\operatorname{Im} \phi(z)||f(z)|^{2} d \mu_{w}(z) \\
& \leq M \int_{G}(\operatorname{Re} \phi(z)-r)|f(z)|^{2} d \mu_{w}(z) \\
& =M \operatorname{Re}\left\langle M_{\phi} f-r f, f\right\rangle_{\mathcal{H}},
\end{aligned}
$$

so that (8) is satisfied. The desired conclusions now follow from Theorem 2.

The linear operator $A$ associated with the sectorial sesquilinear form $\mathcal{F}$ of Proposition 2 is the Friedrichs extension of $M_{\phi}$.

Lemma 1. Let $\left(G^{\prime}, \phi^{\prime}, w^{\prime}\right)$ be a weighted analytic domain and let $(G, \phi, w)$ be its subdomain. Using $H_{r}$ to denote the half-plane $\{z \in \mathcal{C}: \operatorname{Re} z<r\}$, suppose that $\phi(G) \subset H_{r}^{c}$. Also, assume that $\exp \left(-k \phi^{\prime}\right) \in P_{\phi^{\prime}}^{2}\left(G^{\prime}, \mu_{w^{\prime}}\right)$, for every non-negative integer $k$. Let $\omega$ be the unbounded connected component of $\overline{\phi(G)}^{c}$ in $\mathcal{C}$ that contains $H_{r}$, and let $M_{\phi}$ be as described in Proposition 1. If $\phi^{\prime}\left(G^{\prime}\right) \cap H_{r}$ is non-empty, then $\sigma\left(\overline{M_{\phi}}\right) \subset \omega^{c}$; if, in addition, $\overline{\phi(G)}$ is connected, then $\sigma\left(\overline{M_{\phi}}\right)=\overline{\phi(G)}$.

Proof. Assume the hypotheses and also that $\phi^{\prime}\left(G^{\prime}\right) \cap H_{r}$ is non-empty. Suppose that $\sigma\left(\overline{M_{\phi}}\right)$ is not contained in $\omega^{c}$, where $\omega$ is the unbounded connected component of $\overline{\phi(G)}$ c that contains $H_{r}$. The operator $N_{\phi}$ as described in the paragraph preceding Proposition 2 is a normal extension of $M_{\phi}$ and hence of $\overline{M_{\phi}}$. It is easy to check that $\sigma\left(N_{\phi}\right)=\overline{\phi(G)}$. From the proof of Theorem 2 of [9] (see Remark 1 above) one has either $\omega \cap \sigma\left(\overline{M_{\phi}}\right)=\emptyset$ or $\omega \subset \sigma\left(\overline{M_{\phi}}\right)$. Since $\sigma\left(\overline{M_{\phi}}\right)$ is not contained in $\omega^{c}, \omega \subset \sigma\left(\overline{M_{\phi}}\right)$.

Note that $M_{\phi}$ is a cyclic subnormal operator in $\mathcal{H}=P_{\phi}^{2}\left(G, \mu_{w}\right)$ with the cyclic vector 1. With $\gamma\left(M_{\phi}\right)$ as defined in (1), $\sigma\left(\overline{M_{\phi}}\right) \backslash \sigma_{a p}\left(\overline{M_{\phi}}\right) \subset \gamma\left(M_{\phi}\right)$ as recorded in our comments following (1). Since $\sigma_{a p}\left(N_{\phi}\right)=\sigma\left(N_{\phi}\right)=\overline{\phi(G)}$ and $N_{\phi}$ extends $\overline{M_{\phi}}$, we have $\sigma_{a p}\left(\overline{M_{\phi}}\right) \subset \sigma_{a p}\left(N_{\phi}\right)=\overline{\phi(G)}$ and thus $\sigma\left(\overline{M_{\phi}}\right) \backslash \overline{\phi(G)} \subset \gamma\left(M_{\phi}\right)$. Since $\omega \subset \sigma\left(\overline{M_{\phi}}\right)$ and $\omega \cap \overline{\phi(G)}=\emptyset$, we have $\omega \subset \gamma\left(M_{\phi}\right)$. From the observations following (1) in Section 1, we have $\gamma\left(M_{\phi}\right) \subset \operatorname{int}\left(\sigma_{p}\left(M_{\phi}^{*}\right)^{*}\right)$. Since $H_{r} \subset \omega$, one has $H_{r} \subset \sigma_{p}\left(M_{\phi}^{*}\right)^{*}$.

Let $h \in \phi^{\prime}\left(G^{\prime}\right) \cap H_{r}$. Then $\operatorname{Re} h<r \leq \operatorname{Re} \phi(z)$, for every $z$ in $G$, in view of $h \in H_{r}$ and $\phi(G) \subset H_{r}^{c}$. Since $G^{\prime}$ is open and $\phi^{\prime}$ is non-constant analytic, $\phi^{\prime}\left(G^{\prime}\right)$ is open; thus we can choose $\epsilon>0$, for which $h-\epsilon \in \phi^{\prime}\left(G^{\prime}\right) \cap H_{r}$. Consider the function $f: G \rightarrow \mathcal{C}$ defined by $f(z)=1 /(1-\exp (-\phi(z)+h-\epsilon))(z \in G)$. For any integer $m \geq 0$ and for 
any $z \in G$, one has

$$
\begin{aligned}
\left|\sum_{k \geq m+1} \exp (-\phi(z)+h-\epsilon) k\right| & \leq \sum_{k \geq m+1}|\exp \{(-\phi(z)+h-\epsilon) k\}| \\
& =\sum_{k \geq m+1} \exp \{(-\operatorname{Re} \phi(z)+\operatorname{Re} h-\epsilon) k\} \\
& \leq \sum_{k \geq m+1} \exp (-\epsilon k)
\end{aligned}
$$

and the last expression tends to 0 as $m$ tends to $\infty$. Letting $\mathcal{L}=L^{2}\left(G, \mu_{w}\right)$, one has

$$
\begin{aligned}
\left\|f-\sum_{k=0}^{m} \exp \{(-\phi(\cdot)+h-\epsilon) k\}\right\|_{\mathcal{L}}^{2} & =\int_{G}\left|\sum_{k \geq m+1} \exp \{(-\phi(z)+h-\epsilon) k\}\right|^{2} d \mu_{w}(z) \\
& \leq\left(\sum_{k \geq m+1} \exp (-\epsilon k)\right)^{2} \mu_{w}(G) .
\end{aligned}
$$

Since each $\exp \left(-k \phi^{\prime}\right) \in P_{\phi^{\prime}}^{2}\left(G^{\prime}, \mu_{w^{\prime}}\right)$, by assumption, it is clear that $f \in P_{\phi}^{2}\left(G, \mu_{w}\right)$; in fact we have that $\left\|f-\sum_{k=0}^{m} \exp \{(-\phi(\cdot)+h-\epsilon) k\}\right\|_{\mathcal{H}}^{2}$ converges to 0 as $m$ tends to $\infty$. Since $h-\epsilon \in \phi^{\prime}\left(G^{\prime}\right)$, there exists $z_{0} \in G^{\prime}$ such that $\phi^{\prime}\left(z_{0}\right)=h-\epsilon$. Since $H_{r} \subset \sigma_{p}\left(M_{\phi}^{*}\right)^{*}$ and since $h-\epsilon \in H_{r}$, one has $h-\epsilon \in \sigma_{p}\left(M_{\phi}^{*}\right)^{*}$. By Lemma 2 of [9], there exists a constant $c_{h}>0$ such that $|p(h-\epsilon)| \leq c_{h}\left\|p\left(M_{\phi}\right) 1\right\|_{\mathcal{H}}$, for every polynomial $p$. Thus $\left|p\left(\phi^{\prime}\left(z_{0}\right)\right)\right| \leq c_{h}\|p \circ \phi\|_{\mathcal{H}}$, for every polynomial $p$. Since each $\exp \left(-k \phi^{\prime}\right) \in \mathcal{H}^{\prime}=P_{\phi^{\prime}}^{2}\left(G^{\prime}, \mu_{w^{\prime}}\right)$, we can find, for every integer $k \geq 0$, a sequence $\left\{p_{n, k}\right\}_{n \geq 1}$ of polynomials such that $\left\|p_{n, k} \circ \phi^{\prime}-\exp \left\{-k\left(\phi^{\prime}(\cdot)-h+\epsilon\right)\right\}\right\|_{\mathcal{H}^{\prime}}$ converges to 0 as $n$ tends to $\infty$. Since $\mathcal{H}^{\prime} \subset L_{a}^{2}\left(G^{\prime}, \mu_{w^{\prime}}\right)$, there exists, in view of $(10), k_{z_{0}} \in \mathcal{K}^{\prime}=L_{a}^{2}\left(G^{\prime}, \mu_{w^{\prime}}\right)$ such that $\left\langle f, k_{z_{0}}\right\rangle_{\mathcal{K}^{\prime}}=f(z)$ for every $f \in \mathcal{H}^{\prime}$; in particular, using $\phi^{\prime}\left(z_{0}\right)=h-\epsilon$, one obtains $\left\langle p_{n, k} \circ \phi^{\prime}-\exp \left\{-k\left(\phi^{\prime}(\cdot)-h+\epsilon\right)\right\}, k_{z_{0}}\right\rangle_{\mathcal{K}^{\prime}}=p_{n, k}\left(\phi^{\prime}\left(z_{0}\right)\right)-1$, for every $n \geq 1$. Since, for any integer $k \geq 0$,

$$
\begin{aligned}
\left|p_{n, k}\left(\phi^{\prime}\left(z_{0}\right)\right)-1\right| & \leq\left\|p_{n, k} \circ \phi^{\prime}-\exp \left\{-k\left(\phi^{\prime}(\cdot)-h+\epsilon\right)\right\}\right\|_{\mathcal{K}^{\prime}}\left\|k_{z_{0}}\right\|_{\mathcal{K}^{\prime}} \\
& =\left\|p_{n, k} \circ \phi^{\prime}-\exp \left\{-k\left(\phi^{\prime}(\cdot)-h+\epsilon\right)\right\}\right\|_{\mathcal{H}^{\prime}}\left\|k_{z_{0}}\right\|_{\mathcal{K}^{\prime}},\left|p_{n, k}\left(\phi^{\prime}\left(z_{0}\right)\right)-1\right|
\end{aligned}
$$

converges to 0 as $n$ tends to $\infty$. The preceding observations then lead to the following: for every integer $m \geq 0,\left\|\sum_{k=0}^{m} p_{n, k} \circ \phi^{\prime}-\sum_{k=0}^{m} \exp \left\{-k\left(\phi^{\prime}(\cdot)-h+\epsilon\right)\right\}\right\|_{\mathcal{H}^{\prime}}$ and hence $\left\|\sum_{k=0}^{m} p_{n, k} \circ \phi-\sum_{k=0}^{m} \exp \{-k(\phi(\cdot)-h+\epsilon)\}\right\|_{\mathcal{H}}$ converges to 0 and $\sum_{k=0}^{m} p_{n, k}\left(\phi^{\prime}\left(z_{0}\right)\right)$ converges to $m+1$ as $n$ tends to $\infty$. Putting $p=\sum_{k=0}^{m} p_{n, k}$ in $\left|p\left(\phi^{\prime}\left(z_{0}\right)\right)\right| \leq c_{h}\|p \circ \phi\|_{\mathcal{H}}$ and letting $n$ tend to $\infty$, one obtains $m+1 \leq c_{h}\left\|\sum_{k=0}^{m} \exp \{-k(\phi(\cdot)-h+\epsilon)\}\right\|_{\mathcal{H}}$, for every integer $m \geq 0$. This contradicts the fact that $\left\|\sum_{k=0}^{m} \exp \{-k(\phi(\cdot)-h+\epsilon)\}\right\|_{\mathcal{H}}$ converges to $\|f\|_{\mathcal{H}}$ as $m$ tends to $\infty$. Thus we must have $\sigma\left(\overline{M_{\phi}}\right) \subset \omega^{c}$.

Now assume in addition that $\overline{\phi(G)}$ is connected so that $\omega^{c}=\overline{\phi(G)}$. From what we saw above, $\sigma\left(\overline{M_{\phi}}\right) \subset \overline{\phi(G)}$. Let $\lambda \in \phi(G)$; that is, $\lambda=\phi\left(z_{1}\right)$ for some $z_{1}$ in $G$. Suppose $\lambda \in$ $\sigma\left(\overline{M_{\phi}}\right)^{c}$, so that $\overline{M_{\phi}}-\lambda$ is invertible. Then $1 \in\left(\overline{M_{\phi}}-\lambda\right) \mathcal{D}\left(\overline{M_{\phi}}\right)$ and hence there exists $\psi \in \mathcal{D}\left(\overline{M_{\phi}}\right)$ such that $(\phi-\lambda) \psi=1$. Thus $\psi$ cannot be bounded in a neighborhood of $z_{1}$, which contradicts the fact that $\psi$ is analytic on $G$. Hence $\phi(G) \subset \sigma\left(\overline{M_{\phi}}\right)$. Since $\sigma\left(\overline{M_{\phi}}\right)$ is closed, however, $\overline{\phi(G)} \subset \sigma\left(\overline{M_{\phi}}\right)$. 
EXAmPLES 1. (a) Consider the weighted analytic domain $\left(G^{\prime}, \phi^{\prime}, w^{\prime}\right)$, where $G^{\prime}=\mathcal{C}$, $\phi^{\prime}(z)=\sum_{n=0}^{d} a_{n} z^{n}$ with $a_{d} \neq 0$ and $d \geq 1$, and $w^{\prime}(z)=\exp \left(-|z|^{d+\epsilon}\right)$ with $\epsilon>0$. Let $\Sigma$ be any non-empty open subset of $\mathcal{C}$ contained in $\Lambda_{r, M}\left(\subset H_{r}^{c}\right)$. Then $\left(G=\phi^{\prime-1}(\Sigma), \phi=\right.$ $\left.\phi^{\prime} / G, w=w^{\prime} / G\right)$ is a subdomain of $\left(G^{\prime}, \phi^{\prime}, w^{\prime}\right)$. Since $\phi^{\prime}\left(G^{\prime}\right)=\mathcal{C}, \phi^{\prime}\left(G^{\prime}\right) \cap H_{r}=H_{r}$.

(b) Consider the weighted analytic domain $\left(G^{\prime}, \phi^{\prime}, w^{\prime}\right)$, where $G^{\prime}=\mathcal{C} \backslash\{z \in \mathcal{C}$ : $\operatorname{Re} z \leq 0\}, \phi^{\prime}(z)=z^{\alpha}$ with $0<\alpha<1$, and $w^{\prime}(z)=\exp \left(-|z|^{\alpha+\epsilon}\right)$ with $\epsilon>0$. Let $\Sigma$ be any non-empty open subset of $\mathcal{C}$ contained in $\Lambda_{0, M} \cap \phi^{\prime}\left(G^{\prime}\right)$. Then $\left(G=\phi^{\prime-1}(\Sigma), \phi=\right.$ $\left.\phi^{\prime} / G, w=w^{\prime} / G\right)$ is a subdomain of $\left(G^{\prime}, \phi^{\prime}, w^{\prime}\right)$. Note that $\phi^{\prime}\left(G^{\prime}\right) \cap H_{0}$ is non-empty if $\alpha>1 / 2$.

(c) Consider the weighted analytic domain $\left(G^{\prime}, \phi^{\prime}, w^{\prime}\right)$, where $G^{\prime}=\mathcal{C} \backslash\{z \in \mathcal{C}$ : $\operatorname{Re} z \leq 0\}, \phi^{\prime}(z)=\ln z$, and $w^{\prime}(z)=\exp \left(-|z|^{1+\epsilon}\right)$ with $\epsilon>0$. Let $\Sigma$ be any non-empty open subset of $\mathcal{C}$ contained in $\Lambda_{r, M}$. Then $\left(G=\phi^{\prime-1}(\Sigma), \phi=\phi^{\prime} / G, w=w^{\prime} / G\right)$ is a subdomain of $\left(G^{\prime}, \phi^{\prime}, w^{\prime}\right)$. Note that $\phi^{\prime}\left(G^{\prime}\right) \cap H_{r}$ is a non-empty proper subset of $H_{r}$.

In (a), (b) and (c), it can be verified that $\exp \left(-k \phi^{\prime}\right) \in P_{\phi^{\prime}}^{2}\left(G^{\prime}, \mu_{w^{\prime}}\right)$, for every nonnegative integer $k$; thus the conclusion of Lemma 1 holds for Examples 1.

THEOREM 3. Let $\left(G^{\prime}, \phi^{\prime}, w^{\prime}\right)$ be a weighted analytic domain and let $(G, \phi, w)$ be its subdomain. Suppose that $\phi(G)$ is contained in the cone $\Lambda_{r, M}=\{z \in \mathcal{C}:|\operatorname{Im} z| \leq$ $M(\operatorname{Re} z-r)\}$, where $r \in \mathcal{R}$ and $M \in(0, \infty)$, and $\exp \left(-k \phi^{\prime}\right) \in P_{\phi^{\prime}}^{2}\left(G^{\prime}, \mu_{w^{\prime}}\right)$, for every non-negative integer $k$. Let $H_{r}$ be the half-plane $\{z \in \mathcal{C}: \operatorname{Re} z<r\}$. Further, let $M_{\phi}$ be as described in Proposition 1 and let $\phi^{\prime}\left(G^{\prime}\right) \cap H_{r}$ be non-empty. Then the following are true:

(i) the Friedrichs extension $A$ of $M_{\phi}$ is $\overline{M_{\phi}}$; thus $A \subset M_{\phi}^{\max }$;

(ii) $M_{\phi}$ has an analytic model;

(iii) $A=M_{\phi}^{\max }$ provided $\overline{\phi(G)}^{c}$ is connected.

Proof. (i) By Lemma 1, we have $\sigma\left(\overline{M_{\phi}}\right) \subset \omega^{c}$, where $\omega$ is the unbounded connected component of $\overline{\phi(G)}^{c}$ that contains $H_{r}$. Since $A$ is the Friedrichs extension of $M_{\phi}$, $\sigma(A) \subset \Lambda$ as noted earlier (refer to (9)). Since $A$ is closed, $A$ extends $\overline{M_{\phi}}$; further, $\sigma\left(\overline{M_{\phi}}\right) \subset \omega^{c}$ and $\sigma(A) \subset \Lambda$ imply that $\sigma\left(\overline{M_{\phi}}\right)^{c} \cap \sigma(A)^{c}$ is nonempty. It then follows from Lemma 1.6.14 of [6] that $A=\overline{M_{\phi}}$.

(ii) As noted in Proposition $1, \phi(G) \subset \sigma_{p}\left(M_{\phi}^{*}\right)^{*}$; in particular, $\sigma_{p}\left(M_{\phi}^{*}\right) \neq \varnothing$. By Theorem 1 , there exists a surjective partial isometry $W: P_{\phi}^{2}\left(G, \mu_{w}\right) \rightarrow \mathcal{H}_{r}$, where $\mathcal{H}_{r}$ is as described in the paragraph preceding Theorem 1. It suffices then to check that the null space of $W$ is trivial. We adapt the argument of Proposition 9 of [9] to the present situation. Let $f \in P_{\phi}^{2}\left(G, \mu_{w}\right)$ be such that $W f=0$. Examining the proof of Theorem 6 in [9], we find that $W(p \circ \phi)(\lambda)=p(\lambda)$, for any polynomial $p$ and any $\lambda$ in $\sigma_{p}\left(M_{\phi}^{*}\right)^{*}$. Since $f$ is in $\mathcal{H}=P_{\phi}^{2}\left(G, \mu_{w}\right)$, there exists a sequence $p_{n}$ of polynomials such that $\| p_{n} \circ$ $\phi-f \|_{\mathcal{H}}$ converges to 0 . Then there exists a subsequence $\left\{q_{n}\right\}$ of $\left\{p_{n}\right\}$ such that, $z$-almost everywhere $\left[\mu_{w}\right], W\left(q_{n} \circ \phi\right)(\phi(z))=q_{n}(\phi(z))=\left(q_{n} \circ \phi\right)(z)$ converges to $f(z)$. Also, since $W$ is continuous, $\left\|W\left(q_{n} \circ \phi\right)-W f\right\|_{\mathcal{H}_{r}}$ converges to 0 . Since $\mathcal{H}_{r}$ is a reproducing kernel Hilbert space, it follows that $W\left(q_{n} \circ \phi\right)(\phi(z))$ converges to $(W f)(\phi(z))$; thus, $z$-almost everywhere $\left[\mu_{w}\right],(W f)(\phi(z))=f(z)$. Hence $f$ vanishes almost everywhere $\left[\mu_{w}\right]$; but $f$ is analytic so that $f=0$.

(iii) The proof here is an adaptation of the proof of Proposition 11 in [9]. Suppose $\overline{\phi(G)}^{c}$ is connected. By Lemma 1 we have $\sigma\left(\overline{M_{\phi}}\right)=\overline{\phi(G)}$. Further, Proposition 1 yields that $\phi(G) \subset \sigma_{p}\left(M_{\phi}^{*}\right)^{*}$. As recorded in Section 1, $\gamma\left(M_{\phi}\right)$ as defined in (1) is an open subset of $\operatorname{int}\left(\sigma_{p}\left(M_{\phi}^{*}\right)^{*}\right)$ and $\operatorname{int}\left(\sigma_{p}\left(M_{\phi}^{*}\right)^{*}\right) \backslash \gamma\left(M_{\phi}\right)$ is a nowhere dense subset of $\mathcal{C}$. Therefore $\gamma\left(M_{\phi}\right)$ is non-empty. By part (ii) the cyclic subnormal operator $M_{\phi}$ has an 
analytic model, and it follows from Theorem 9 of [9] that $\gamma\left(M_{\phi}\right)=\sigma\left(\overline{M_{\phi}}\right) \backslash \sigma_{a p}\left(\overline{M_{\phi}}\right)=$ $\overline{\phi(G)} \backslash \sigma_{a p}\left(\overline{M_{\phi}}\right)$. Since $\gamma\left(M_{\phi}\right)$ is a non-empty open set, it is easy to see that there exists $\lambda_{0} \in \gamma\left(M_{\phi}\right) \cap\left(\phi(G) \backslash \sigma_{q p}\left(\overline{M_{\phi}}\right)\right)$. Let $f \in \mathcal{D}\left(M_{\phi}^{\max }\right)$; then $\left(M_{\phi}^{\max }-\lambda_{0}\right) f \in \mathcal{H}=$ $P_{\phi}^{2}\left(G, \mu_{w}\right)$. Thus there exists a sequence $\left\{q_{n} \circ \phi\right\}$ such that $\left\|q_{n} \circ \phi-\left(M_{\phi}^{\max }-\lambda_{0}\right) f\right\|_{\mathcal{H}}$ converges to 0 . Let $z_{0} \in G$ be such that $\phi\left(z_{0}\right)=\lambda_{0}$. By $(10),\left(q_{n} \circ \phi\right)\left(z_{0}\right)$ converges to 0 ; consequently, $t_{n}=q_{n} \circ \phi-\left(q_{n} \circ \phi\right)\left(z_{0}\right) \in \operatorname{lin}\left\{\phi^{k}: k=0,1,2, \ldots\right\}, t_{n}$ converges to $\left(M_{\phi}^{\max }-\lambda_{0}\right) f$, and $t_{n}$ vanishes at $z_{0}$ for all $n$. Let $p_{n} \in \operatorname{lin}\left\{\phi^{k}: k=0,1,2, \ldots\right\}$ be such that $t_{n}=\left(\phi-\phi\left(z_{0}\right)\right) p_{n}$. Since $\lambda_{0}$ is not in $\sigma_{a p}\left(\overline{M_{\phi}}\right)$, there exists a positive number $N$ such that $\left\|\left(\overline{M_{\phi}}-\lambda_{0}\right) h\right\|_{\mathcal{H}} \geq N\|h\|_{\mathcal{H}}$ for every $h \in \mathcal{D}\left(\overline{M_{\phi}}\right)$. Putting $h=p_{n}$ one has $\left\|t_{n}\right\|_{\mathcal{H}} \geq N\left\|p_{n}\right\|_{\mathcal{H}}$, for all $n$. This shows that the sequence $\left\{p_{n}\right\}$ converges to some $g$ in $\mathcal{H}$. Since $\left(M_{\phi}-\lambda_{0}\right) p_{n}=t_{n}$ converges to $\left(M_{\phi}^{\max }-\lambda_{0}\right) f$, the closedness of $\overline{M_{\phi}}$ shows that $g \in \mathcal{D}\left(\overline{M_{\phi}}\right)$ and $\left(M_{\phi}^{\max }-\lambda_{0}\right) f=\left(\overline{M_{\phi}}-\lambda_{0}\right) g$. This in turn implies that $f=g$ except possibly at $z_{0}$; but both $f$ and $g$ are analytic so that $f=g$. Thus $f \in \mathcal{D}\left(\overline{M_{\phi}}\right)$, showing that $\mathcal{D}\left(M_{\phi}^{\max }\right) \subset \mathcal{D}\left(\overline{M_{\phi}}\right)$.

COROLLARY 1. Under the hypotheses of Theorem 3 and assuming $\overline{\phi(G)}^{c}$ to be connected, one has that, for any $f \in \mathcal{D}\left(M_{\phi}^{\max }\right)$, there exists a sequence $p_{n} \circ \phi$ of polynomials in $\phi$ such that $p_{n} \circ \phi$ converges to $f$ in $P_{\phi}^{2}\left(G, \mu_{w}\right)$ and $\phi\left(p_{n} \circ \phi\right)$ converges to $\phi f$ in $P_{\phi}^{2}\left(G, \mu_{w}\right)$.

In the light of Theorem 3, it would be desirable to construct an example of a weighted analytic domain $(G, \phi, w)$ for which $\overline{M_{\phi}} \neq M_{\phi}^{\max }$. One could for that purpose try to use the weighted analytic domains $\left(G^{\prime}, \phi^{\prime}, w^{\prime}\right)$ and subdomains $(G, \phi, w)$ mentioned in Examples 1; indeed, the examples discussed there allow for $\overline{\phi(G)}$ c to be disconnected. We have, however, not been able to construct such an example.

Question 1. Does there exist a weighted analytic domain $(G, \phi, w)$ for which $\overline{M_{\phi}} \neq M_{\phi}^{\max }$ ?

3. Applications: Galerkin approximation and generalized wave equation. Our first application of Theorem 3 is a Galerkin approximation result in the setting of $P_{\phi}^{2}\left(G, \mu_{w}\right)$ corresponding to a weighted analytic domain $(G, \phi, w)$. See Sections 2.8 and 2.12 of [6].

Proposition 3. Let $\left(G^{\prime}, \phi^{\prime}, w^{\prime}\right)$ be a weighted analytic domain and let $(G, \phi, w)$ be its subdomain. Suppose that $\phi(G)$ is contained in the cone $\Lambda_{r, M}=\{z \in \mathcal{C}:|\operatorname{Im} z| \leq$ $M(\operatorname{Re} z-r)\}$ where $r \in \mathcal{R}, M \in(0, \infty)$ and $\exp \left(-k \phi^{\prime}\right) \in P_{\phi^{\prime}}^{2}\left(G^{\prime}, \mu_{w^{\prime}}\right)$, for every nonnegative integer $k$. Let $H_{r}$ be the half-plane $\{z \in \mathcal{C}: \operatorname{Re} z<r\}$, and assume that $\phi^{\prime}\left(G^{\prime}\right) \cap$ $H_{r}$ is non-empty. For $n \geq 1$, let $v_{n}=\operatorname{lin}\left\{\phi_{1}, \ldots, \phi_{n}\right\}$, where $\phi_{k}=\phi^{k-1}(k \geq 1)$ and let $\lambda \in \mathcal{C}$ be such that $\operatorname{Re} \lambda<r$. If $f \in P_{\phi}^{2}\left(G, \mu_{w}\right)$ then, for each $n \geq 1$, there exists a unique $f_{n}$ in $v_{n}$ such that

$$
\int_{G}(\phi(z)-\lambda) f_{n}(z) \overline{\phi^{k-1}(z)} d \mu_{w}(z)=\int_{G} f(z) \overline{\phi^{k-1}(z)} d \mu_{w}(z)(1 \leq k \leq n) .
$$

Moreover,

$$
\begin{aligned}
& \lim _{n \rightarrow \infty} \int_{G}\left|f_{n}(z)-\frac{f(z)}{(\phi(z)-\lambda)}\right|^{2} d \mu_{w}(z) \\
& \quad \leq \lim _{n \rightarrow \infty} \int_{G}(\operatorname{Re} \phi(z)-r+1)\left|f_{n}(z)-\frac{f(z)}{(\phi(z)-\lambda)}\right|^{2} d \mu_{w}(z)=0 .
\end{aligned}
$$


Proof. Let $\mathcal{H}=P_{\phi}^{2}\left(G, \mu_{w}\right)$ and let $S=M_{\phi}$ in $\mathcal{H}$ with $\mathcal{D}(S)=\cup_{n \geq 1} v_{n}=\operatorname{lin}\left\{\phi^{k}: k=\right.$ $0,1,2, \ldots\}$. The condition $\phi(G) \subset \Lambda_{r, M}=\{z \in \mathcal{C}:|\operatorname{Im} z| \leq M(\operatorname{Re} z-r)\}$ guarantees, as in the proof of Proposition 2, that $\left|\operatorname{Im}\langle S f, f\rangle_{\mathcal{H}}\right| \leq M \operatorname{Re}\langle S f-r f, f\rangle_{\mathcal{H}}$, for all $f$ in $\mathcal{D}(S)$. An appeal to Theorem 2.12.6 of [6] now yields (11). Since the Friedrichs extension $A$ of $S$ is $\overline{M_{\phi}}$ in view of Theorem 3, one more appeal to Theorem 2.12.6 of [6] yields (12) in view of part (b) of Proposition 2.

EXAMPLES 2. Referring to (a) of Examples 1, consider the weighted analytic domain $\left(G^{\prime}, \phi^{\prime}, w^{\prime}\right)$, where $G^{\prime}=\mathcal{C}, \phi^{\prime}(z)=z$, and $w^{\prime}(z)=\exp \left(-|z|^{2}\right)$; (that is, $d \mu_{w^{\prime}}$ is the Gaussian measure). Then $\left(G=\operatorname{int}\left(\Lambda_{1, M}\right), \phi=\phi^{\prime} / G, w=w^{\prime} / G\right)$ is a subdomain of $\left(G^{\prime}, \phi^{\prime}, w^{\prime}\right)$. Note that $\exp \left(-k \phi^{\prime}\right) \in P_{\phi^{\prime}}^{2}\left(G^{\prime}, \mu_{w^{\prime}}\right)$, for every non-negative integer $k$. Then, for any $f \in P_{\phi}^{2}\left(G, \mu_{w}\right)$, there exists a sequence of polynomials $p_{n}$ with the degree of $p_{n}$ not bigger than $n$ such that

$$
\lim _{n \rightarrow \infty} \int_{G}\left|p_{n}(z)-\frac{f(z)}{z}\right|^{2} d \mu_{w}(z)=0
$$

and

$$
\lim _{n \rightarrow \infty} \int_{G}(\operatorname{Re} z)\left|p_{n}(z)-\frac{f(z)}{z}\right|^{2} d \mu_{w}(z)=0 .
$$

Our second application of Theorem 3 is obtaining a 'smooth' $P_{\phi}^{2}\left(G, \mu_{w}\right)$-valued solution of a generalized wave equation. (See Section 4.4 of [6].) For that purpose we establish a lemma.

LEMMA 2. Let $(G, \phi, w)$ be a weighted analytic domain and let $\phi(G)$ be contained in $\Lambda_{r, M} \cap P$, where $\Lambda_{r, M}=\{z \in \mathcal{C}:|\operatorname{Im} z| \leq M(\operatorname{Re} z-r)\}$ and $P=\left\{z \in \mathcal{C}:(\operatorname{Im} z)^{2} \leq\right.$ $M(\operatorname{Re} z-r+1)\}$ with $r \in \mathcal{R}$ and $M \in(0, \infty)$. Let $\mathcal{F}$ be the sesquilinear sectorial form defined on $\Gamma$ and let $A$ be the associated operator (that is, the Friedrichs extension of $M_{\phi}$ in $\left.\mathcal{H}=P_{\phi}^{2}\left(G, \mu_{w}\right)\right)$ as guaranteed by Proposition 2. Then the following are true:

(i) $|\mathcal{F}(f, g)-\overline{\mathcal{F}(g, f)}| \leq 2 M^{1 / 2}\|f\|_{\Gamma}\|g\|_{\mathcal{H}}$, for all $f, g \in \Gamma$;

(ii) $\Gamma=\mathcal{D}\left((A-a)^{1 / 2}\right)$, where $a=r-1$.

Proof. (i) Using the condition $\phi(G) \subset \Lambda_{r, M} \cap P$ one has, for any $f, g \in \Gamma$,

$$
\begin{aligned}
|\mathcal{F}(f, g)-\overline{\mathcal{F}(g, f)}| & =\mid \int_{G} \phi(z) f(z) \overline{g(z)} d \mu_{w}-\overline{\int_{G} \phi(z) g(z) \overline{f(z)} d \mu_{w} \mid} \\
& =\left|\int_{G}(\phi(z)-\overline{\phi(z)}) f(z) \overline{g(z)} d \mu_{w}\right| \\
& \leq 2 \int_{G}|\operatorname{Im} \phi(z)\|f(z)\| g(z)| d \mu_{w} \\
& \leq 2\left(\int_{G}|\operatorname{Im} \phi(z)|^{2}|f(z)|^{2} d \mu_{w}(z)\right)^{1 / 2}\left(\int_{G}|g(z)|^{2} d \mu_{w}(z)\right)^{1 / 2} \\
& \leq 2 M^{1 / 2}\|f\|_{\Gamma}\|g\|_{\mathcal{H}} .
\end{aligned}
$$

(ii) The desired result follows from part (i) above, Theorem 2.8.12 and Corollary 6.1.14 of [6] and the choice $a=r-1$ as recorded at the end of Section 1 . 
For a Hilbert space $\mathcal{H}$ and for a positive integer $k$, let $C^{k}([0, \infty), \mathcal{H})$ denote the vector space of $\mathcal{H}$-valued $k$ times continuously differentiable functions on $[0, \infty)$. For $u \in C^{k}([0, \infty), \mathcal{H})$ and for a positive integer $m \leq k$, let $u^{(m)}(t)$ denote the $m$ th derivative of $u$ at $t$.

Proposition 4. Let $\left(G^{\prime}, \phi^{\prime}, w^{\prime}\right)$ be a weighted analytic domain and let $(G, \phi, w)$ be its subdomain. Also, suppose that $\phi(G)$ is contained in $\Lambda_{r, M} \cap P$, where $\Lambda_{r, M}=\{z \in$ $\mathcal{C}:|\operatorname{Im} z| \leq M(\operatorname{Re} z-r)\}$ and $P=\left\{z \in \mathcal{C}:(\operatorname{Im} z)^{2} \leq M(\operatorname{Re} z-r+1)\right\}$ with $r \in \mathcal{R}$ and $M \in(0, \infty)$. Let $\exp \left(-k \phi^{\prime}\right) \in P_{\phi^{\prime}}^{2}\left(G^{\prime}, \mu_{w^{\prime}}\right)$, for every non-negative integer $k$. Let $H_{r}$ be the half-plane $\{z \in \mathcal{C}: \operatorname{Re} z<r\}$, and assume that $\phi^{\prime}\left(G^{\prime}\right) \cap H_{r}$ is non-empty. If $A$ is the Friedrichs extension of $M_{\phi}$ as guaranteed by Proposition 2 and $\Gamma$ is as in part (ii) of Lemma 2, then for each $f \in \mathcal{D}(A), g \in \Gamma$ there exists a unique $u \in C^{2}\left([0, \infty), P_{\phi}^{2}\left(G, \mu_{w}\right)\right) \cap$ $C^{1}([0, \infty), \Gamma)$ such that $u(0)=f, u^{(1)}(0)=g, u(t) \in \mathcal{D}(A)$ for all $t \in[0, \infty)$, and $u^{(2)}(t)=$ $-\phi u(t)$, for all $t \in[0, \infty)$.

Proof. As shown, by Theorem 3, the action of the Friedrichs extension $A$ of $M_{\phi}$ is multiplication by $\phi$. The desired conclusion now follows from Lemma 2 above and Theorem 4.4 .2 of [6].

It should be clear to the reader that all of the examples mentioned previously can be suitably modified so as to make the hypotheses of Proposition 4 applicable to them.

\section{REFERENCES}

1. N. Aronszajn, Theory of reproducing kernels, Trans. Amer. Math. Soc. 68 (1950), 337 404.

2. A. Athavale and S. Chavan, Sectorial forms and unbounded subnormals, preprint.

3. J. B. Conway, The theory of subnormal operators, Mathematical Surveys and Monographs, Vol. 36 (Amer. Math. Soc., Providence, 1991).

4. D. E. Edmonds and W. D. Evans, Spectral theory and differential operators, Oxford Science Publications (Clarendon Press, Oxford, 1987).

5. T. Kato, Perturbation theory for linear operators (Springer-Verlag, 1984).

6. Milan Miklavčič, Applied functional analysis and partial differential equations (World Scientific, Singapore, 1998).

7. J. Stochel and F. Szafraniec, On normal extensions of unbounded operators, I, J. Operator Theory 14 (1985), 31-55.

8. J. Stochel and F. Szafraniec, On normal extensions of unbounded operators, II, Acta Sci. Math. (Szeged) 53 (1989), 153-177.

9. J. Stochel and F. Szafraniec, On normal extensions of unbounded operators, III. Spectral properties, Publ. RIMS, Kyoto Univ. 25 (1989), 105-139. 